\section{ORIGINAL RESEARCH}

\author{
C.P. Geer
}

J. Simonds

A. Anvery

M.Y. Chen

J.H. Burdette

M.E. Zapadka

T.L. Ellis

S.B. Tatter

G.J. Lesser

M.D. Chan

K.P. McMullen

A.J. Johnson

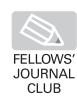

\title{
Does MR Perfusion Imaging Impact Management Decisions for Patients with Brain Tumors? A Prospective Study
}

BACKGROUND AND PURPOSE: MR perfusion imaging can be used to help predict glial tumor grade and disease progression. Our purpose was to evaluate whether perfusion imaging has a diagnostic or therapeutic impact on clinical management planning in patients with glioma.

MATERIALS AND METHODS: Standard MR imaging protocols were interpreted by a group of 3 NRs in consensus, with each case being interpreted twice: first, including routine sequences; and second, with the addition of perfusion imaging. A multidisciplinary team of treating physicians assessed tumor status and created hypothetical management plans, on the basis of clinical presentation and routine MR imaging and then routine MR imaging plus perfusion MR imaging. Physicians' confidence in the tumor status assessment and management plan was measured by using Likert-type items.

RESULTS: Fifty-nine consecutive subjects with glial tumors were evaluated; 50 had known pathologic diagnoses. NRs and the treatment team agreed on tumor status in $45 / 50$ cases $(\kappa=0.81)$. With the addition of perfusion, confidence in status assessment increased in $20(40 \%)$ for NRs and in $28(56 \%)$ for the treatment team. Of the 59 patient-care episodes, the addition of perfusion was associated with a change in management plan in 5 (8.5\%) and an increase in the treatment team's confidence in their management plan in $34(57.6 \%)$. NRs and the treatment team found perfusion useful in most episodes of care and wanted perfusion included in future MR images for $>80 \%$ of these subjects.

CONCLUSIONS: Perfusion imaging appears to have a significant impact on clinical decision-making and subspecialist physicians' confidence in management plans for patients with brain tumor.

ABBREVIATIONS: $\mathrm{ASL}=$ arterial spin-labeled; $\mathrm{COE}=$ Center of Excellence; $\mathrm{DSC}=$ dynamic susceptibility contrast; GBM = glioblastoma multiforme; $N R=$ neuroradiologist; PASL = pulsed arterial spin-labeling; ROC = receiver operating characteristic; SPGR = spoiled gradient-recalled

G liomas remain the most common malignant primary brain tumor in adults. ${ }^{1}$ As improving second- and thirdline therapies for treating gliomas become available, ${ }^{2-4}$ it is increasingly important to be able to predict tumor progression at earlier stages when patients maintain a performance status such that they can tolerate further therapies. It is also critical to accurately assess which gliomas on follow-up imaging are progressing on a current treatment regimen so that therapy can be modified appropriately. Given that standard therapy for highgrade glial tumors usually includes radiation as well as temozolomide, ${ }^{5,6}$ enhancing lesions associated with edema on follow-up imaging in patients with brain tumor can often represent either a treatment-related effect (eg, necrosis, pseudoprogression) or tumor progression, depending on the relative timing of therapy. ${ }^{7}$ In fact, imaging response criteria have changed recently because of the inability of conventional MR imaging to accurately predict tumor progression, and new re-

\section{Received April 27; accepted after revision June 13.}

From the Departments of Radiology (C.P.G., A.A., M.Y.C., J.H.B., M.E.Z., J.S., A.J.J.); Radiology, School of Medicine (J.S.); Neurosurgery (C.P.G.; S.B.T., T.L.E.); Internal Medicine, Hematology/Oncology Section (G.J.L.); and Radiation Oncology (K.P.M., M.D.C.), Wake Forest University Health Sciences, Wake Forest Baptist Health, Winston-Salem, North Carolina.

Paper previously presented in part at: 48th Annual Meeting of the American Society of Neuroradiology, May 14-20, 2010; Boston, Massachusetts.

Please address correspondence to Annette J. Johnson, MD, MS, Department of Radiology, Wake Forest Baptist Health, Medical Center Blvd, Winston-Salem, NC 27157; e-mail: anjohnso@wfubmc.edu

http://dx.doi.org/10.3174/ajnr.A2811 sponse criteria suggest allowing a 25\% increase in tumor size before determining tumor progression by imaging. ${ }^{8}$

Recent studies have indicated that perfusion MR imaging, both evaluation of relative CBV by using a DSC technique and CBF by using a PASL technique, can help predict the grade of astrocytoma and the likelihood of rapid progression. ${ }^{9-30}$ Some studies have also suggested that perfusion imaging may be helpful in differentiating treatment-related effects versus tumor progression. ${ }^{11,16,31-33}$ These studies have suggested that MR perfusion imaging performs reasonably well as a diagnostic test in this setting. Given this evidence for diagnostic accuracy, a logical next step is to evaluate the extent to which having this perfusion imaging in a clinical setting affects the care of patients with glial tumors - that is, its diagnostic and therapeutic impact in patients with glial tumors. ${ }^{34}$

The goal of the study was to determine whether an experienced multidisciplinary team of subspecialist physicians would find perfusion MR imaging useful in a clinical setting. In particular, we sought to evaluate whether the addition of perfusion imaging to routine MR imaging would significantly impact the intermediate outcome of treatment planning, including confidence in tumor status and a specific management plan.

\section{Materials and Methods}

The study was reviewed and approved by our institutional review board, which deemed it Health Insurance Portability and Accountability Act-compliant and waived the requirement for informed consent. Study subjects included consecutive adults (outpatients and in- 
patients) with either biopsy-proven glioma or imaging findings most consistent with glial tumor (in the absence of pathology) who were imaged at our center from September 2007 through May 2008. Since September 2007, all patients with a known or suspected intracranial mass have been imaged by using a brain tumor MR imaging protocol that includes either DSC or PASL or both (depending on scanner limitations). An individual patient was included in the study only once, at the point in time of their reference MR imaging (reflecting an episode of their care at a single point in time). Names of all patients undergoing brain tumor protocol MR imaging were acquired from the radiology information system records during a 2-year period. All patients' electronic medical records were reviewed by investigators (A.A., A.J.J.) who were not involved in the subsequent image interpretation or treatment planning. Patients with a known diagnosis (at the time of the reference MR imaging) of oligodendroglial tumor ( $n=$ 10) were excluded because of known limitations in predicting tumor grade with perfusion. ${ }^{13,27}$ Four patients were excluded because a treatment-team member recognized the patient, and 1 was excluded because of very poor quality images.

\section{Design}

This was a prospective study evaluating the effect of the addition of perfusion MR imaging on hypothetical management plans (an intermediate outcome), in which the cases were acquired in a retrospective manner. After identification of the cases, the MR images were prospectively interpreted with a standardized reporting format (the NR report) by 3 NRs in consensus (years of experience: 13, 3, and 3). The NR report included both categorical (multiple choice and openended) and 10-point Likert-type items. The NRs were provided with all available relevant clinical information at the time of the reference MR imaging (eg, any previous biopsy results, treatment history, current symptoms). Each patient's reference brain MR imaging was interpreted twice by the NR group, always in the same order and always compared with the patient's prior MR imaging if extant. First, the examination was interpreted after a review of the routine sequences (T1-, T2-weighted, FLAIR, diffusion-weighted, and postcontrast T1weighted and SPGR); second (immediately thereafter), after review of the routine sequences plus the perfusion MR imaging. Perfusion maps were qualitatively assessed by comparing the mass lesions with normal-appearing white matter in the same image.

Hypothetical management plans were also determined prospectively. Each patient scenario was presented to the existing multidisciplinary treatment team of physicians who specialize in the care of patients with brain tumor at our institutional Brain Tumor COE. In clinical practice, the team treats an average of 250 new patients with brain tumors per year. For the study, this team, functioning within the National Cancer Institute-designated Comprehensive Cancer Center, always included a neuro-oncologist (16 years of experience), 1-2 neurosurgeons (10 and 14 years of experience), and 1-2 radiation oncologists (2.5 and 7 years of experience). The team meets weekly to discuss clinical patients, including formal reviews of treatment history, the latest MR imaging results, and current medical status, and to discuss treatment options and formulate a consensus plan.

For the study, the treatment team was asked to provide detailed hypothetical treatment plans in a standardized format (the neurooncology treatment plan). The neuro-oncology treatment plan included both categorical (multiple choice and open-ended) and 10point Likert-type items. The treatment team was provided with all of the relevant clinical information as well as the NR report during the group's usual weekly clinical conference, to closely simulate the group's actual practice in the care of patients with brain tumors. For the study, the team was asked to indicate several parameters at the point in care of the reference MR imaging: tumor status (choices included improved, stable, or progressed), confidence in this estimation of tumor status (10-point Likert-type item), chosen management plan (categorical), and confidence in this chosen management plan (10-point Likert-type item). Both the NRs and the treatment team were asked to indicate the usefulness of the perfusion imaging in each particular patient at that snapshot in time and whether they would want perfusion imaging included in the future for that particular patient. The neuro-oncology treatment plan was completed twice for each patient: first, on the basis of routine MR imaging and the NR report of the routine MR imaging; and second, on the basis of the perfusion MR imaging and the NR report of the perfusion MR imaging. Both the NR group and the treatment team were also asked whether they found DSC or PASL more useful in each case.

\section{Imaging}

MR images on patients in the study were performed with either 1.5T Signa scanners or 3T Signa Excite scanners (GE Healthcare, Milwaukee, Wisconsin). Conventional MR images were acquired by using the following protocol: 3-plane localizer, sagittal T1-weighted spin-echo imaging, coronal fast FLAIR imaging, and axial fast T2-weighted imaging. Contrast-enhanced 3D SPGR, T1-weighted coronal and axial postcontrast spin-echo images were also obtained.

Our PASL technique, including methods for acquisition, labeling, and postprocessing, has been previously described. ${ }^{35}$ This method includes single subtraction with thin section $\mathrm{TI}_{1}$ periodic saturation ${ }^{36}$ with a flow-sensitive alternating inversion recovery ${ }^{37,38}$; postprocessing involves subtraction of tag/control image pairs, motion correction, segmentation of the anatomic T1-weighted image, and voxelwise computation of CBF maps, which are then colored by using a standard scale and the resultant JPEG of the map sent to the PACS. ${ }^{35}$

The DSC images were acquired with a gradient echo-planar imaging sequence during the second pass of a standard-dose $(0.1 \mathrm{mmol}$ per $\mathrm{kg}$ ) bolus of gadopentetate dimeglumine (Magnevist; Bayer Schering Pharma, Berlin, Germany). Eleven to 16 axial sections were positioned to cover the tumor on the basis of the T2-weighted and FLAIR images. Imaging parameters were as follows: FOV, $230 \times 230$ $\mathrm{mm}$; section thickness, $5 \mathrm{~mm}$ on $1.5 \mathrm{~T}$ and $7 \mathrm{~mm}$ on $3 \mathrm{~T}$; matrix, $128 \times$ 128; flip angle, $35^{\circ}$; and band width, $250 \mathrm{kHz}$. Contrast was injected at a rate of $4 \mathrm{~mL}$ per second, with one-third being administered as a loading dose and the remaining two-thirds being administered as a bolus for acquisition of a gradient-echo echo-planar T2* DSC sequence. DSC maps were created by MR imaging technologists by using the scanner or an Advantage workstation using FuncTool (GE Healthcare; semiautomated), with color-map images being sent to the PACS.

\section{Analysis}

The primary outcome variable was management plan at the point in time of care of the reference MR imaging. Results were analyzed by using statistical software (StatView, Version 4.5, 1996; Abacus Concepts, Berkeley, California). Analyses were performed by using descriptive statistics. Likert-type items for confidence were anchored at 1 for least confidence and 10 for greatest confidence. For each patient, direct comparisons were made between confidence in the tumor status and confidence in the treatment plan with routine MR imaging versus perfusion MR imaging. The effect of the MR imaging type on these parameters was evaluated by using the Wilcoxon signed rank 


\begin{tabular}{llccc}
\hline \multicolumn{2}{l}{$\begin{array}{l}\text { Table 1: Tumor status estimations for subjects by NRs and the } \\
\text { treatment team }\end{array}$} \\
\hline & & \multicolumn{3}{c}{ NRs } \\
\cline { 2 - 4 } & Improved & Stable & Progressed \\
\hline Treatment team & Improved & $3(6)$ & & \\
& Stable & $1(2)$ & $27(54)$ & $3(6)$ \\
& Progressed & & $1(2)$ & $15(30)$ \\
\hline
\end{tabular}

${ }^{a}$ Total cases $(N=50)$. Numbers in parentheses are percentages.

Table 2: Effect of perfusion imaging on confidence in determination of tumor status ${ }^{\mathrm{a}}$

\begin{tabular}{lccc}
\hline & $\begin{array}{c}\text { Increased } \\
\text { Confidence }\end{array}$ & $\begin{array}{c}\text { No Change in } \\
\text { Confidence }\end{array}$ & $\begin{array}{r}\text { Decreased } \\
\text { Confidence }\end{array}$ \\
\hline NRs & $20(40)$ & $24(48)$ & $6(12)$ \\
Treatment team & $28(56)$ & $12(24)$ & $10(20)$ \\
\hline
\end{tabular}

${ }^{a} N=50$ cases with previous tissue diagnoses. Numbers in parentheses are percentages.

test (treating the 10-point ratings as ordinal). Effect of the MR imaging type on the management plan was evaluated by the McNemar test. $P$ values $<.05$ were considered statistically significant.

\section{Results}

A total of 59 consecutive adult patients with suspected glial tumors were evaluated, including 27 women and 32 men. Evaluations were performed separately by the NRs and the treatment team, at multiple sessions over 11 months. Among the 50 cases with known pathologic diagnoses from previous biopsy or resection, a wide spectrum of glial tumor histology was represented, with 6 grade I, 9 grade II, 10 grade III, and 25 grade IV glial tumors. Of the 9 patients who were initially presenting at the reference time of evaluation (ie, were treatment-naïve and had no previous biopsy), 7 had a subsequent tissue diagnosis of GBM, 1 had grade II astrocytoma, and 1 was treated presumptively as having a low-grade glioma (brain stem mass). Of the 59 patients, 2 were on antiangiogenic therapy at the time of the study.

Both the NRs and the treatment team of physicians were asked to indicate each patient's tumor status at the point in time of the reference MR imaging, with status choices including improved, stable, or progressed (Table 1). The NRs and the treatment team agreed on the tumor status in 45/50 episodes of care $(\kappa=0.81)$. The addition of perfusion imaging had a significant effect on the confidence rating for tumor status for both NRs $(P<.007)$ and the treatment team $(P<.04$; Wilcoxon signed rank test). Table 2 details the type of effect the addition of perfusion images had on the NRs' and treatment team's confidence in the tumor status.

In $5 / 59(8.5 \%)$ patient-care episodes, the management plan changed on the basis of the addition of perfusion imaging to the routine sequences (Table 3). An example is shown in
Fig 1. Two of the 5 were cases in which confidence in tumor status decreased with the addition of perfusion images. Although the study was undertaken to evaluate the intermediate outcome of the impact on the management plan and not the effectiveness of the plan itself (ie, there was retrospective acquisition of patients, and hypothetical prospective treatment plans were created months to years after the actual treatment of these patients and therefore had no effect on their actual care), we undertook further poststudy chart review of these 5 patients to try to ascertain whether these changes in hypothetical treatment plans based on perfusion results would have been appropriate for these patients at the referenced point in time, given the actual subsequent course of their disease. In the patient in whom perfusion results led to a change in the hypothetical plan from biopsy to follow-up MR imaging (patient A in Table 3), the lesion on subsequent scanning 4 months later had not progressed and the MR imaging appearance was presumed at that time to be treatment effect. In the 2 patients in whom perfusion results led to a change from routine to shorter interval follow-up MR imaging (patients B and E), the first patient progressed through additional temozolomide treatment at the time of the reference MR imaging and then further progressed through the addition of an etoposide 4 months later and then elected hospice care; the second patient died 10 days after the reference MR imaging in acute renal failure. In the patient in whom perfusion results led to a change from routine follow-up MR imaging to re-resection (patient $\mathrm{C}$ ), the next MR imaging at our institution 6 months later showed convincing evidence of further tumor progression and there was subsequent re-resection, which confirmed progression. In the patient in whom perfusion results led to a change from short-term follow-up MR imaging to enrollment in a therapeutic trial (patient D), the patient began additional temozolomide treatment at the time of the reference MR imaging but 3 months later had further progression and was started on an investigational trial.

Each member of the treatment team of physicians was also asked to rate their confidence in the team's chosen management plan at the care episode occurring at the time of the reference MR imaging. The addition of perfusion imaging had a significant effect on the team's confidence rating for their management plan $(P<.0001$, Wilcoxon signed rank test). Figure 2 shows the type of effect that the addition of perfusion images had on the treatment team's confidence in their chosen management plan.

Of the treatment-naïve patients evaluated at the time of initial clinical presentation (ie, with no previous tissue diagnosis), $1 / 9(11.1 \%)$ had a change in the suspected diagnosis based on areas of increased perfusion within the lesion, changing the suspected diagnosis from likely low-grade to likely

\begin{tabular}{|c|c|c|c|c|}
\hline Patient & $\begin{array}{l}\text { Patient Age } \\
\text { (yr)/Sex }\end{array}$ & Previous Pathology & Effect of Perfusion & Change in Management Plan \\
\hline A & $54 / \mathrm{M}$ & GBM & Confidence in tumor progression decreased & Cancelled biopsy and short-interval follow-up MR \\
\hline B & $56 / M$ & Grade II astrocytoma & Confidence in tumor progression increased & Shorter interval to follow-up MRI \\
\hline C & $51 / \mathrm{M}$ & Grade II astrocytoma & Confidence in tumor progression increased & Routine follow-up MRI changed to re-resection \\
\hline D & $51 / F$ & GBM & Confidence in tumor progression increased & $\begin{array}{l}\text { Short-term follow-up MRI changed to enrollment } \\
\text { in a therapeutic trial }\end{array}$ \\
\hline$E$ & $55 / M$ & GBM & Confidence in stable disease decreased & Shorter interval to follow-up MRI \\
\hline
\end{tabular}



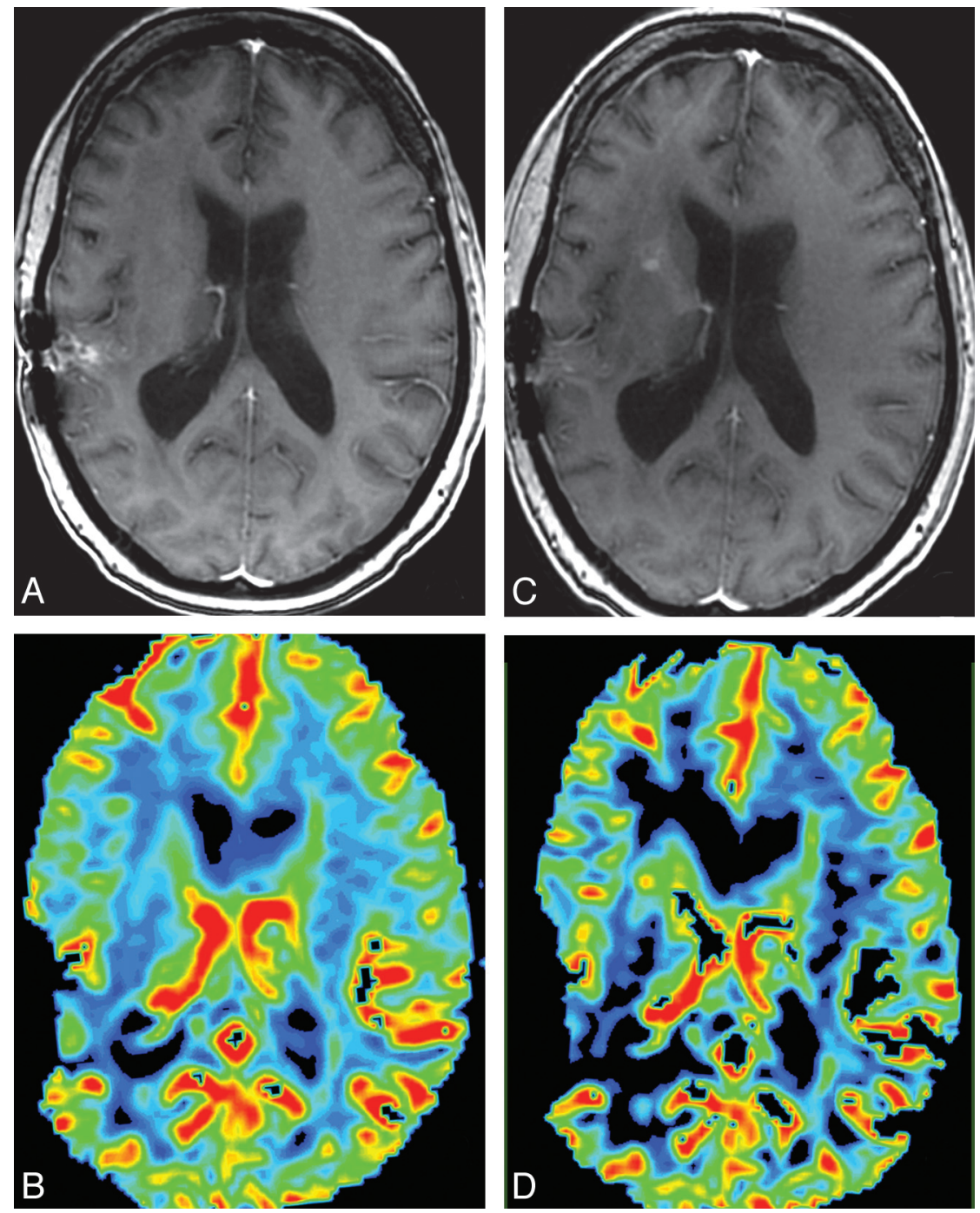

Fig 1. $A$ and $C$, Postcontrast SPGR image for reference MRI $(C)$ shows new enhancement along the right anterior limb of the internal capsule, which was not present on comparison postcontrast SPGR $(A)$. $B$ and $D$, This area shows increased CBV on the concurrent DSC color map $(D)$ compared with the prior DSC color map $(B)$. The appearance was most suggestive of tumor progression, and the management plan was altered accordingly. Susceptibility artifacts are seen along the previous resection cavity in the right temporoparietal region on all images.

\section{Confidence in treatment plan}

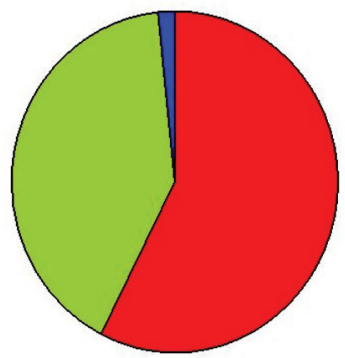

\section{$\square$ Increased $\square$ Stable $\square$ Decreased}

Fig 2. Pie chart shows the effect of the addition of perfusion imaging on treatment-team confidence in their chosen management plan.

high-grade glial tumor of the brain stem. On subsequent poststudy chart review, this 23-year-old patient later underwent stereotactic biopsy with scant tissue most consistent with grade II astrocytoma and was treated with 54 Gy radiation. Follow-up imaging at 6.5 months showed a stable pontine lesion with development of a second $\mathrm{T} 2$ hyperintense lesion within her upper cervical cord; both lesions were stable 6 months later.

Among these patients with brain tumor, both the NRs and the treatment team found perfusion imaging to be useful (ie, $>5$ on the 10-point Likert-type scale) in more than half of these episodes of care. Both NRs and the treatment team wanted future MR imaging in these specific patients to include perfusion imaging (Fig 3).

In 6 cases, only PASL was performed, and in 6 cases, only DSC was performed. In 47 cases, both DSC and PASL were performed with the reference MR imaging. In these 47 cases, NRs and the treatment team found either or both perfusion sequences to be useful in $41(87.2 \%)$ and 38 (80.9\%), respectively. Among these cases, both NRs and the treatment team indicated variable relative usefulness of the specific perfusion sequence (Fig 4). NRs found neither type of perfusion sequence to be useful in $6(12.8 \%)$, and the treatment team 


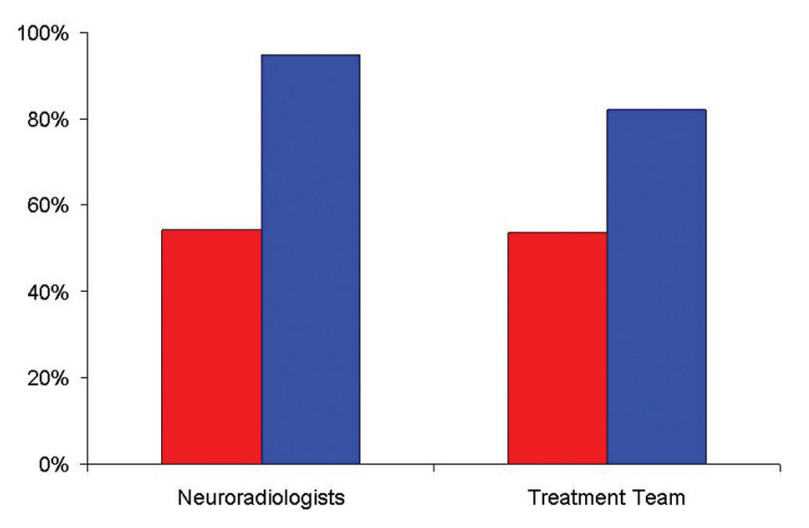

口Perfusion useful in this episode $\square$ Want perfusion in future MRIs for this patient

Fig 3. Bar graph shows the frequency of the perceived usefulness of perfusion imaging in these study subjects.

\section{Perfusion sequence types which were thought more useful}

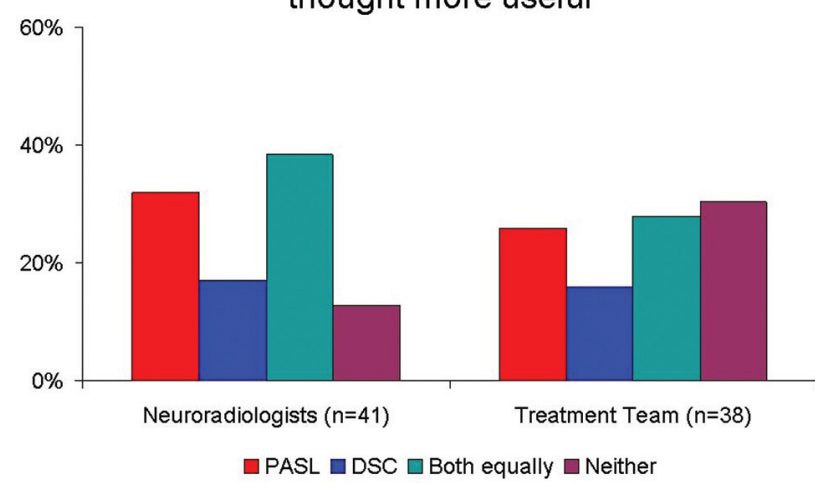

Fig 4. Bar graph shows the relative frequencies that specific perfusion techniques were thought most useful by NRs and the treatment team.

found neither to be useful in $9(19.1 \%)$ of these care episodes. Among these cases for the NRs, 5/6 showed no changes at all on conventional sequences, and 1/6 showed decreased enhancement and $\mathrm{T} 2$ hyperintensity in a patient known to be on bevacizumab. Among these cases for the treatment team, 8/9 showed no changes on conventional sequences and $1 / 9$ was treatment naïve without pathologic diagnosis.

\section{Discussion}

Recent studies have indicated that relative CBV and CBF associated with primary glial tumors of the CNS can be used to predict tumor grade and the likelihood of progression. ${ }^{2-23}$ Studies have suggested that perfusion MR imaging performs reasonably well as a diagnostic test in the setting of treated patients, in that high-grade gliomas typically have relatively increased $\mathrm{CBF}$ and $\mathrm{CBV}$ and treatment-related effects are associated with a relative decrease in $\mathrm{CBF}$ and CBV. ${ }^{11,16,31-33,39}$ However, to our knowledge, there is a paucity of literature regarding the extent to which these perfusion MR imaging techniques might actually affect treatment decisions in a clinical setting - that is, whether they have diagnostic or therapeutic impact. In particular, it has been unclear whether a group of experienced physicians who specialize in the treatment of patients with brain tumor on a daily basis would actually use perfusion imaging or find it helpful in clinical decision-making. In our study, we presented information related to single episodes of care, including clinical histories and current symptoms for 59 consecutive patients with gliomas to NRs for prospective scan interpretation, without and with perfusion imaging. Then we presented the same information and NR reports of current MR imaging results for these patients to the physicians in our multidisciplinary Brain Tumor COE treatment team for evaluation. The team's task was to formulate a consensus management plan by using this information, first without and then with the perfusion imaging. While focused on and experienced in the care of patients with brain tumor, the team members were not very experienced with perfusion imaging and were at the outset of the study fairly skeptical as to the clinical usefulness of perfusion imaging in this setting, in particular, whether perfusion MR imaging would offer additional useful information over conventional MR imaging.

The study results-particularly the finding of increased treatment-team confidence in the management plan in 57.6\% of these patient episodes-suggest that even experienced and highly specialized clinical neuro-oncologic experts will often find perfusion imaging helpful in the commonly complex management decisions in patients with glial tumors. This result is not surprising given evidence that CBV or CBF measures or both have been seen in other recent (largely retrospective) studies to correlate with tumor grade and, in high-grade glial tumors, with cellular proliferation, response to treatment, and time to progression. ${ }^{12,13,26,27,29,30,32,39-42}$ In the current study, not only was the experts' confidence significantly affected by the addition of the perfusion imaging, but the management plan was actually changed in 5 of the 59 patient episodes of care $(8.5 \%)$. Although this was not a diagnosticaccuracy study, we subsequently retrospectively reviewed these patients' medical records and found that the changes suggested by the perfusion imaging interpretations seemed to have aligned with the subsequent behavior of their tumors in 4 patients ( 1 patient's subsequent tumor behavior could not be determined).

If approximately $8.5 \%$ of episodes of care for these patients are potentially affected by the inclusion of perfusion data and if we assume that a patient with a glioma is likely to be imaged approximately every 3-6 months, we suggest that perfusion imaging has a good chance of affecting clinical management for these patients at some time during the course of their disease. Given this potential for diagnostic and therapeutic impact, we suggest that it should, therefore, be considered as a standard part of their imaging. Our rationale is that perfusion imaging is noninvasive and can be included in the brain MR imaging that is already part of a patient's regular follow-up. Even if both types of perfusion scans are obtained, they add only approximately 11 minutes to the examination time. Individual scanner limitations, rather than throughput constraints, were the primary reason that we did not perform both PASL and DSC on all of these patients.

DSC is more widely accepted for the evaluation of perfusion in brain tumors. However, given the variable perceived usefulness of each technique in this study and published early evidence that ASL could possibly offer slightly greater accuracy in distinguishing tumor progression from radiation-induced changes, ${ }^{43}$ we continue to do both sequences in patients with brain tumor. Our overarching goal in this technology application is to provide patient-centered care. We think that 
the required additional scanning time, postprocessing time (by our technologists or in an automated process), and interpretation time with these data would likely be considered worthwhile to most patients, given that the data have a reasonable chance of increasing the confidence of their physicians in determining their tumor status and management plan. Nevertheless, the effect on workflow is a concern for a busy neuroradiologic practice-which is a major motivator for our choosing to use qualitative rather than quantitative analysis of the imaging data.

The limitations of this study include its relatively small size and retrospective acquisition of cases. While there was some risk that NRs or physicians on the treatment team recalled specific patients (ie, from prior actual treatment) included in the study, we believe that the risk of this potential bias is small, because we delayed the onset of the study for $>14$ months after the latest reference MR imaging and included a question on each report and neuro-oncology treatment plan specifically asking whether participants recalled the patient in each case on the basis of the history or images. The prospective creation of management plans that are only hypothetical may also be considered a relative weakness of the study. However, given the aim of the study, we suggest that the design is not a weakness but a strength. The study was neither a test of diagnostic accuracy nor a prospective trial undertaken to affect patient care. It was designed to focus on other necessary aspects of the technologic assessment of a diagnostic test, namely the diagnostic and therapeutic impact. If a diagnostic test does not have an effect on management decisions, it would be difficult to argue that the test could be very useful in clinical care. The chosen design has been successfully used in other studies that evaluated the impact of imaging technologies on intermediate outcomes. ${ }^{44,45}$ Whether perfusion imaging has a positive effect on patient outcome was not studied with this design.

The study is limited in that we evaluated only a single episode of care for these patients, who are typically seen repeatedly during the course of their disease; we do not know whether perfusion data acquired over time will be useful in an individual patient with glial tumor. Given the expertise of all the physicians involved in the study and the referral pattern to our Brain Tumor COE, our results may not be generalizable to non-tertiary care settings, sites without a COE for brain tumor treatment, or settings where NRs are not experienced with qualitative assessment of perfusion imaging.

Before the performance of the study, the involved physicians had several years of experience in observing the quality and typical appearances of the qualitative perfusion maps we produce for hundreds of clinical brain tumor cases. The cause of the observed decrease in confidence in tumor status in a minority of cases is not known. Two of these cases led to treatment plan changes. We subsequently informally reviewed all of the other cases in which confidence decreased with the addition of perfusion images, and we found that common features included nearby susceptibility artifacts, small lesions, or low confidence levels with the routine MR imaging interpretations. It may be that closer imaging follow-up would be prudent whenever the addition of perfusion imaging leads to a decrease in confidence in tumor status.

Not all of our study cases had both DSC and PASL performed, so we are cautious in drawing any conclusions about which type of perfusion technique may be perceived as more useful. Cases in which neither perfusion sequence technique was thought useful tended to be those in which the conventional sequences had shown no change at all since the prior study. Finally, most published studies on the topic of MR perfusion for brain tumors have used quantitative analyses, so our qualitative analysis in this study may be considered a limitation. We know of no study directly comparing qualitative versus quantitative analysis of DSC perfusion data (though we are undertaking such a study currently), but a study by Kim and $\mathrm{Kim}^{19}$ suggested that the ROC curve analyses showed no significant difference between quantitative and qualitative analyses with PASL. Because in our clinical practice, we qualitatively analyze perfusion maps (and judge that quantitative analysis on a routine basis is impractical given our volume and workflow), we deliberately sought to determine whether our typical evaluation of these data would impact the intermediate outcome of the management plan. We sought to examine the effectiveness, rather than the efficacy, of perfusion as we use the data in our current practice. Whether quantitative analysis of these data would prove more or less useful is not answered with this design.

\section{Conclusions}

The addition of perfusion imaging to routine MR imaging in patients with glial tumors appears to impact NRs' and treating physicians' confidence in tumor status as well as the treatment team's choice of a management plan and the confidence in that plan. Experts in the diagnosis and treatment of patients with brain tumors find perfusion MR imaging with qualitative analysis helpful in a clinical setting and want it in the future for these patients.

Disclosures: Stephen Tatter-UNRELATED: Board Membership: Monteris Medical, Comments: Medical Advisory Board 2002-2009 for this company, which manufactures a laser for interstitial thermal therapy of brain tumors. Glenn Lesser-UNRELATED: Consultancy. Merck Schering-Plough; Payment for Lectures, including Service on Speakers Bureaus: Prostrakan. Michael D. Chan-UNRELATED: Payment for Lectures, including Service on Speakers Bureaus: Elekta, Comments: received honorarium for 1-time lecture at Eletka users' meeting.

\section{References}

1. Central Brain Tumor Registry of the United States. CBTRUS Statistical Report: primary brain and central nervous system tumors diagnosed in the United States in 2004-2007. 2011. http://www.cbtrus.org. Accessed 2011 Feb 21.

2. Gutin PH, Iwamoto FM, Beal K, et al. Safety and efficacy of bevacizumab with hypofractionated stereotactic irradiation for recurrent malignant gliomas. Int J Radiat Oncol Biol Phys 2009;26:156-63. Epub 2009 Jan 23

3. Reardon DA, Fink KL, Mikkelsen T, et al. Randomized phase II study of cilengitide, an integrin-targeting arginine-glycine-aspartic acid peptide, in recurrent glioblastoma multiforme. J Clin Oncol 2008;26:5610-17. Epub 2008 Nov 3

4. Vredenburgh JJ, Desjardins A, Herndon JE 2nd, et al. Phase II trial of bevacizumab and irinotecan in recurrent malignant glioma. Clin Cancer Res 2007: $13 ; 1253-59$

5. Fiveash JB, Spencer SA. Role of radiation therapy and radiosurgery in glioblastoma multiforme. Cancer J 2003;9:222-29

6. Stupp R, Mason WP, van den Bent MJ, et al. Radiotherapy plus concomitant and adjuvant temozolomide for glioblastoma. N Engl J Med 2005;352:987-96

7. Kumar AJ, Leeds NE, Fuller GN, et al. Malignant gliomas: MR imaging spectrum of radiation therapy- and chemotherapy-induced necrosis of the brain after treatment. Radiology 2000;217:377-84

8. Wen PY, Macdonald DR, Reardon DA, et al. Updated response assessment criteria for high-grade gliomas: response assessment in neuro-oncology working group. J Clin Oncol 2010;28:1963-72. Epub 2010 Mar 15

9. Aronen HJ, Gazit IE, Louis DN, et al. Cerebral blood volume maps of gliomas: comparison with tumor grade and histologic findings. Radiology 1994;191: $41-51$ 
10. Aronen HJ, Glass J, Pardo FS, et al. Echo-planar MR cerebral blood volume mapping of gliomas: clinical utility. Acta Radiol 1995;36:520-28

11. Aronen HJ, Perkio J Dynamic susceptibility contrast MRI of gliomas. Neuroimaging Clin N Am 2002;12:501-23

12. Barajas RF Jr, Hodgson JG, Chang JS, et al. Glioblastoma multiforme regional genetic and cellular expression patterns: influence on anatomic and physiologic MR imaging. Radiology 2010;254:564-76

13. Bisdas S, Kirkpatrick M, Giglio P, et al. Cerebral blood volume measurements by perfusion-weighted MR imaging in gliomas: ready for prime time in predicting short-term outcome and recurrent disease? AJNR Am J Neuroradiol 2009;30:681-88. Epub 2009 Jan 29

14. Cha S. Update on brain tumor imaging: from anatomy to physiology. AJNR Am J Neuroradiol 2006;27:475-87

15. Cha S, Knopp EA, Johnson G, et al. Intracranial mass lesions: dynamic contrast-enhanced susceptibility-weighted echo-planar perfusion MR imaging. Radiology 2002;223:11-29

16. Covarrubias DJ, Rosen BR, Lev MH. Dynamic magnetic resonance perfusion imaging of brain tumors. Oncologist 2004;9:528-37

17. Deibler AR, Pollock JM, Kraft RA, et al. Arterial spin-labeling in routine clinical practice. Part 3. Hyperperfusion patterns. AJNR Am J Neuroradiol 2008; 29:1428-35. Epub 2008 Mar 20

18. Jackson A, Kassner A, Annesley-Williams D, et al. Abnormalities in the recirculation phase of contrast agent bolus passage in cerebral gliomas: comparison with relative blood volume and tumor grade. AJNR Am J Neuroradiol 2002;23:7-14

19. Kim HS, Kim SY. A prospective study on the added value of pulsed arterial spin-labeling and apparent diffusion coefficients in the grading of gliomas. AJNR Am J Neuroradiol 2007;28:1693-99. Epub 2007 Sep 20

20. Kim MJ, Kim HS, Kim JH, et al. Diagnostic accuracy and interobserver variability of pulsed arterial spin labeling for glioma grading. Acta Radiol 2008;49: $450-57$

21. Knopp EA, Cha S, Johnson G, et al. Glial neoplasms: dynamic contrast-enhanced T2*-weighted MR imaging. Radiology 1999;211:791-98

22. Law M, Oh S, Babb JS, et al. Low-grade gliomas: dynamic susceptibilityweighted contrast-enhanced perfusion MR imaging-prediction of patient clinical response. Radiology 2006;238:658-67. Epub 2006 Jan 5

23. Law M, Oh S, Johnson G, et al. Perfusion magnetic resonance imaging predicts patient outcome as an adjunct to histopathology: a second reference standard in the surgical and nonsurgical treatment of low-grade gliomas. Neurosurgery 2006;58:1099-107

24. Law M, Yang S, Babb JS, et al. Comparison of cerebral blood volume and vascular permeability from dynamic susceptibility contrast-enhanced perfusion MR imaging with glioma grade. AJNR Am J Neuroradiol 2004;25:746-55

25. Law $\mathrm{M}$, Yang $\mathrm{S}$, Wang $\mathrm{H}$, et al. Glioma grading: sensitivity, specificity, and predictive values of perfusion MR imaging and proton MR spectroscopic imaging compared with conventional MR imaging. AJNR Am J Neuroradiol 2003;24:1989-98

26. Law M, Young RJ, Babb JS, et al. Gliomas: predicting time to progression or survival with cerebral blood volume measurements at dynamic susceptibility-weighted contrast-enhanced perfusion MR imaging. Radiology 2008;247: 490-98. Epub 2008 Mar 18

27. Lev MH, Ozsunar Y, Henson JW, et al. Glial tumor grading and outcome prediction using dynamic spin-echo MR susceptibility mapping compared with conventional contrast-enhanced MR: confounding effect of elevated rCBV of oligodendrogliomas [corrected]. AJNR Am J Neuroradiol 2004;25:214-21

28. Lev MH, Rosen BR. Clinical applications of intracranial perfusion MR imaging. Neuroimaging Clin N Am 1999;9:309-31

29. Shin JH, Lee HK, Kwun BD, et al. Using relative cerebral blood flow and volume to evaluate the histopathologic grade of cerebral gliomas: preliminary results. AJR Am J Roentgenol 2002;179:783-89
30. Warmuth C, Gunther M, Zimmer C. Quantification of blood flow in brain tumors: comparison of arterial spin labeling and dynamic susceptibilityweighted contrast-enhanced MR imaging. Radiology 2003;228:523-32. Epub 2003 Jun 20

31. Barajas RF Jr, Chang JS, Segal MR, et al. Differentiation of recurrent glioblastoma multiforme from radiation necrosis after external beam radiation therapy with dynamic susceptibility-weighted contrast-enhanced perfusion MR imaging. Radiology 2009;253:486-96. Epub 2009 Sep 29

32. Hu LS, Baxter LC, Smith KA, et al. Relative cerebral blood volume values to differentiate high-grade glioma recurrence from posttreatment radiation effect: direct correlation between image-guided tissue histopathology and localized dynamic susceptibility-weighted contrast-enhanced perfusion MR imaging measurements. AJNR Am J Neuroradiol 2009;30:552-58. Epub 2008 Dec 4

33. Sugahara T, Korogi Y, Tomiguchi S, et al. Posttherapeutic intraaxial brain tumor: the value of perfusion-sensitive contrast-enhanced MR imaging for differentiating tumor recurrence from nonneoplastic contrast-enhancing tissue. AJNR Am J Neuroradiol 2000;21:901-09

34. Hollingworth W, Jarvik JG. Technology assessment in radiology: putting the evidence in evidence-based radiology. Radiology 2007;244:31-38. Epub 2007 May 23

35. Deibler AR, Pollock JM, Kraft RA, et al. Arterial spin-labeling in routine clinical practice. Part 1. Technique and artifacts. AJNR Am J Neuroradiol 2008;29: 1228-34. Epub 2008 Mar 27

36. Luh WM, Wong EC, Bandettini PA, et al. QUIPSS II with thin-slice TI1 periodic saturation: a method for improving accuracy of quantitative perfusion imaging using pulsed arterial spin labeling. Magn Reson Med 1999;41:1246-54

37. Kim SG, Tsekos NV. Perfusion imaging by a flow-sensitive alternating inversion recovery (FAIR) technique: application to functional brain imaging. Magn Reson Med 1997;37:425-35

38. Kim SG, Tsekos NV, Ashe J. Multi-slice perfusion-based functional MRI using the FAIR technique: comparison of CBF and BOLD effects. NMR Biomed 1997;10:191-96

39. Kong DS, Kim ST, Kim EH, et al. Diagnostic dilemma of pseudoprogression in the treatment of newly diagnosed glioblastomas: the role of assessing relative cerebral blood flow volume and oxygen-6-methylguanine-DNA methyltransferase promoter methylation status. AJNR Am J Neuroradiol 2011;32:382-87. Epub 2011 Jan 20

40. Jain R, Gutierrez J, Narang J, et al. In vivo correlation of tumor blood volume and permeability with histologic and molecular angiogenic markers in gliomas. AJNR Am J Neuroradiol 2011;32:388-94. Epub 2010 Nov 11

41. Nabors LB, Mikkelsen T, Rosenfeld SS, et al. Phase I and correlative biology study of cilengitide in patients with recurrent malignant glioma. J Clin Onco 2007;25:1651-57

42. Price SJ, Green HA, Dean AF, et al. Correlation of MR relative cerebral blood volume measurements with cellular density and proliferation in high-grade gliomas: an image-guided biopsy study. AJNR Am J Neuroradiol 2011;32:50106. Epub 2010 Dec 16

43. Ozsunar Y, Mullins ME, Kwong K, et al. Glioma recurrence versus radiation necrosis? A pilot comparison of arterial spin-labeled, dynamic susceptibility contrast enhanced MRI, and FDG-PET imaging. Acad Radiol 2010;17:282-90. Epub 2010 Jan 12

44. Johnson AJ, Ying J, El Gammal T, et al. Which MR imaging sequences are necessary in determining the need for radiation therapy for cord compression? A prospective study. AJNR Am J Neuroradiol 2007;28:32-37

45. Yin Y, Evanoff BA, Gilula LA, et al. Surgeons' decision making in patients with chronic wrist pain: role of bilateral three-compartment wrist arthrographyprospective study. Radiology 1996;200:829-32 